MATHEMATICS OF COMPUTATION

Volume 70, Number 233 , Pages $297-306$

S 0025-5718(00)01231-X

Article electronically published on February 23, 2000

\title{
FAST CONVERGENCE OF QUASI-MONTE CARLO FOR A CLASS OF ISOTROPIC INTEGRALS
}

\author{
A. PAPAGEORGIOU
}

\begin{abstract}
We consider the approximation of $d$-dimensional weighted integrals of certain isotropic functions. We are mainly interested in cases where $d$ is large. We show that the convergence rate of quasi-Monte Carlo for the approximation of these integrals is $O(\sqrt{\log n} / n)$. Since this is a worst case result, compared to the expected convergence rate $O\left(n^{-1 / 2}\right)$ of Monte Carlo, it shows the superiority of quasi-Monte Carlo for this type of integral. This is much faster than the worst case convergence, $O\left(\log ^{d} n / n\right)$, of quasi-Monte Carlo.
\end{abstract}

\section{INTRODUCTION}

The Monte Carlo method (MC) is frequently used for multidimensional integration. The expected error of MC, using $n$ integrand evaluations, is of order $n^{-1 / 2}$ independent of the dimension. However, this convergence is not fast, and a large number of evaluations may be necessary.

Quasi-Monte Carlo (QMC) methods evaluate the integrand at deterministic points in contrast to MC methods, which use random points. The deterministic points are, roughly speaking, uniformly spread because they belong to low discrepancy sequences. The Koksma-Hlawka inequality states that the worst case QMC error for multivariate integration is of order $\log ^{d} n / n$, where $n$ is the number of integrand evaluations and $d$ is the dimension. A similar bound for the average error of multidimensional integration is shown by Woźniakowski [18. Niederreiter 6], and Drmota and Tichy 3] are authoritative references on low discrepancy sequences, their properties, and their applications to numerical integration.

The concern about the QMC error is that $\log ^{d} n / n$ becomes huge when $n$ is fixed and $d$ is large as sometimes happens in practice. This has contributed to the belief that QMC methods should not be used for high-dimensional problems [1]. However, tests by Paskov and Traub 12 and Paskov 13 showed that QMC methods can be very effective for high-dimensional integrals arising in computational finance. They used QMC methods to approximate 360-dimensional integrals required for pricing a collateralized mortgage obligation. Other papers reporting the success of QMC methods for problems in finance include [4, 7, 10]. A survey of the state of the art may be found in Chapter 4 of the monograph by Traub and Werschulz [17.

Received by the editor March 2, 1999.

2000 Mathematics Subject Classification. Primary 65D30, 65D32.

Key words and phrases. Multidimensional integration, quadrature, Monte Carlo methods, low discrepancy sequences, quasi-Monte Carlo methods.

This research has been supported in part by the NSF. 
One of the hypotheses advanced to explain the success of QMC methods is that the financial problems are nonisotropic since some dimensions can be far more important than others. In a recent paper, Sloan and Woźniakowski [14] used this fact to obtain a possible theoretical explanation for the surprisingly good performance of QMC methods for problems in finance.

Papageorgiou and Traub [11] used QMC-GF, a QMC method using points from the generalized Faure1 sequence [15, for a model isotropic problem suggested by a physicist B. Keister [5. Their tests on high dimensional instances of this problem ( $d$ ranging from 25 to 100) showed the superiority of QMC-GF over MC and over Keister's proposed quadrature rules. Tests on the same problem by Novak et al. 9] showed that QMC-GF performs extremely well compared to NEW [8], an interpolatory algorithm for multidimensional integration of smooth functions.

In this paper we prove that the worst case speed of convergence of QMC for a class of isotropic functions (which includes the ones tested in 11]) is of order $\sqrt{\log n} / n$. Thus, QMC has two advantages over MC for this class of integrals:

- QMC converges as $\sqrt{\log n} / n$ while MC converges as $n^{-1 / 2}$.

- The worst case error of QMC is $O(\sqrt{\log n} / n)$ while only the expected error of $\mathrm{MC}$ is $O\left(n^{-1 / 2}\right)$.

We summarize the remainder of this paper. For the reader's benefit we briefly list certain properties of low discrepancy sequences in the second section. The problem is formulated in the third section, and fast convergence is proven in the last section.

\section{LOW DISCREPANCY SEQUENCES}

Discrepancy is a measure of deviation from uniformity of a sequence of points. In particular, the discrepancy of $n$ points $x_{1}, \ldots, x_{n} \in[0,1]^{d}, d \geq 1$, is defined by

$$
D_{n}^{(d)}=D^{(d)}\left(x_{1}, \ldots, x_{n}\right)=\sup _{E}\left|\frac{A(E ; n)}{n}-\lambda(E)\right|
$$

where the supremum is taken over all the subsets of $[0,1]^{d}$ of the form $E=$ $\left[0, t_{1}\right) \times \cdots \times\left[0, t_{d}\right), 0 \leq t_{j} \leq 1,1 \leq j \leq d, \lambda$ denotes the Lebesgue measure, and $A(E ; n)$ denotes the number of the $x_{j}$ that are contained in $E$. A detailed analysis of low discrepancy sequences can be found in [3, 6, 15] and in the references therein.

A sequence $x_{1}, x_{2}, \ldots$ of points in $[0,1]^{d}$ is a low discrepancy sequence if

$$
D_{n}^{(d)} \leq c(d) \frac{(\log n)^{d}}{n}, \quad \forall n>1
$$

where the constant $c(d)$ depends only on the dimension $d$.

The Koksma-Hlawka inequality establishes a relation between low discrepancy sequences and multivariate integration (see [6]). If $f$ is a real function defined on $[0,1]^{d}$ of bounded variation $V(f)$ in the sense of Hardy and Krause, then for any sequence $x_{1}, \ldots, x_{n} \in[0,1)^{d}$ we have

$$
\left|\int_{[0,1]^{d}} f(x) d x-\frac{1}{n} \sum_{i=1}^{n} f\left(x_{i}\right)\right| \leq V(f) D_{n}^{(d)} .
$$

\footnotetext{
1 The generalized Faure and the Sobol' low discrepancy sequences are included in FINDER, a Columbia University software system, and are available to researchers upon request by writing the author.
} 
So far, we have discussed the discrepancy of a sequence of points with respect to the Lebesgue measure. We briefly discuss the case where the uniformity of a sequence is assessed with regard to a probability measure $\mu$ and introduce some notation that we will use later. We assume that the support of $\mu$ is $\mathbb{R}_{+}$and that $\mu$ is absolutely continuous with respect to the Lebesgue measure.

For $n>1$, let $x_{i} \in \mathbb{R}_{+}, i=1, \ldots, n$, be any given points. Define the difference between the empirical distribution (approximating $\mu$ using the points $x_{i}$ ) and the measure $\mu$ by

$$
R_{\mu}(E)=\frac{A(E ; n)}{n}-\mu(E), \quad E \subset \mathbb{R}_{+},
$$

where $A(E ; n)$ denotes the number of $x_{i}$ contained in $E$ and does not depend on $\mu$ but depends only on the points $x_{i}$. The discrepancy of the points $x_{i}, i=1, \ldots, n$, with respect to the probability measure $\mu$ is defined by

$$
D_{\mu, n}=D_{\mu, n}\left(x_{1}, \ldots, x_{n}\right)=\sup _{E}\left|R_{\mu}(E)\right|,
$$

where the supremum is taken over all sets of the form $E=[0, x), x \in \mathbb{R}_{+}$.

For $x \geq 0$ we use the following notation:

$$
\begin{aligned}
\mu(x) & =\mu([0, x)), \\
R_{\mu}(x) & =\frac{\sum_{i=1}^{n} 1_{[0, x)}\left(x_{i}\right)}{n}-\mu(x)=\frac{\sum_{i=1}^{n} 1_{[0, \mu(x))}\left(\mu\left(x_{i}\right)\right)}{n}-\mu(x),
\end{aligned}
$$

where $1_{A}$ denotes the characteristic function of a set $A$. Thus, given a low discrepancy sequence (with respect to the Lebesgue measure) $t_{i} \in[0,1], i=1,2, \ldots$, the sequence $x_{i}=\mu^{-1}\left(t_{i}\right) \in \mathbb{R}_{+}, i=1,2, \ldots$, has discrepancy $D_{\mu, n}$, with respect to the measure $\mu$, and satisfies $D_{\mu, n}\left(x_{1}, \ldots, x_{n}\right)=D_{n}^{(1)}\left(t_{1}, \ldots, t_{n}\right), n>1$. For brevity, when $d=1$ we will write $D_{n}$ instead of $D_{n}^{(1)}$.

\section{Problem formulation}

We consider the approximation of a weighted high-dimensional integral of the form

$$
I_{d}(f)=\int_{\mathbb{R}^{d}} f(\|x\|) e^{-\|x\|^{2}} d x
$$

where $d$ is the dimension, $f: \mathbb{R} \rightarrow \mathbb{R}$, and $\|\cdot\|$ denotes the Euclidean norm in $\mathbb{R}^{d}$. We also assume that $f$ is such that the integral (1) is well defined and $f^{\prime}$ exists a.e.

The integral (1) can be reduced, via a change of variable, to a one-dimensional integral, which can often be solved analytically, e.g., $f=$ cos. We do not do this because we want to assess the performance of QMC methods for $d$-dimensional integration. In [11], the empirical convergence rate of QMC is proportional to $n^{-1}$, as if it sees that this is really a one-dimensional problem. In contrast, the empirical convergence rate of MC remains proportional to $n^{-1 / 2}$; it does not see that the problem is really one dimensional. 
We obtain an equivalent integral over the cube $[0,1]^{d}$. We have $(2)$

$$
\begin{aligned}
I_{d}(f) & =\int_{\mathbb{R}^{d}} f(\|x\|) e^{-\|x\|^{2}} d x=2^{-d / 2} \int_{\mathbb{R}^{d}} f(\|y\| / \sqrt{2}) e^{-\|y\|^{2} / 2} d y \\
& =\pi^{d / 2} \int_{\mathbb{R}^{d}} f(\|y\| / \sqrt{2}) \frac{e^{-\|y\|^{2} / 2}}{(2 \pi)^{d / 2}} d y=\pi^{d / 2} \int_{[0,1]^{d}} f\left(\sqrt{\sum_{j=1}^{d}\left(\phi^{-1}\right)^{2}\left(t_{j}\right) / 2}\right) d t
\end{aligned}
$$

where $\phi$ is the cumulative normal distribution function with mean 0 and variance 1 ,

$$
\phi(u)=\frac{1}{\sqrt{2 \pi}} \int_{-\infty}^{u} e^{-s^{2} / 2} d s, \quad u \in[-\infty, \infty] .
$$

Let $t_{i}=\left(t_{i 1}, \ldots, t_{i d}\right) \in[0,1]^{d}, i=1, \ldots, n$, be any deterministically chosen sample points. Let $x_{i}=\left(x_{i 1}, \ldots, x_{i d}\right) \in \mathbb{R}^{d}$, be such that $x_{i j}=\phi^{-1}\left(t_{i j}\right), j=$ $1, \ldots, d, i=1, \ldots, n$. We approximate the integral (1) by the QMC method

$$
I_{d, n}(f)=\frac{\pi^{d / 2}}{n} \sum_{i=1}^{n} f\left(\left\|x_{i}\right\| / \sqrt{2}\right) .
$$

We derive the error equation and the convergence rate of the method $I_{d, n}$ for the following class of functions.

Definition 1. $F$ is the class of functions $f: \mathbb{R} \rightarrow \mathbb{R}$, such that $I_{d}(f)<\infty$, $f$ is absolutely continuous, $f^{\prime}$ exists a.e., and

$$
\text { ess } \sup \left\{\left|f^{\prime}(r)\right|: r \in \mathbb{R}\right\} \leq M,
$$

where $M$ is a constant.

The examples originally considered by Keister [2, 5] and later by Papageorgiou and Traub [11, and Novak et al. 9] belong to $F$ since $f=$ cos. The example $f(r)=\left(1+r^{2}\right)^{1 / 2}$ in [2, 9] also belongs to $F$.

\section{Speed of CONVERGEnCE}

In this section we derive the error and the convergence rate of the method (3) for the integral (1) in the class $F$. We have

$$
\begin{aligned}
I_{d}(f) & =2^{-d / 2} \int_{\mathbb{R}^{d}} f(\|x\| / \sqrt{2}) e^{-\|x\|^{2} / 2} d x \\
& =c_{d} 2^{-d / 2} \int_{0}^{\infty} f(r / \sqrt{2}) r^{d-1} e^{-r^{2} / 2} d r \\
& =\pi^{d / 2} \int_{0}^{\infty} f(r / \sqrt{2}) \mu^{\prime}(r) d r,
\end{aligned}
$$

where $c_{d}=2 \pi^{d / 2} / \Gamma(d / 2)$ and $\mu^{\prime}(\cdot)$ is the density function of the distribution $\mu$ of $r=\|x\|, x \in \mathbb{R}^{d}$. Note that $r^{2}=\|x\|^{2}$ follows the chi-square distribution (see [16] for the relationship between $\mu$ and the chi-square distribution).

First we consider integrals with Gaussian weights and derive the error of a method that uses the average of $n$ function evaluations, at arbitrary points, to approximate them. Then we show the error of the method $I_{d, n}$ in the class $F$, and derive certain auxiliary inequalities for the measure $\mu$. We conclude the section by 
showing that for a particular choice of the $n$ sample points the convergence of the method $I_{d, n}$ is $O(\sqrt{\log n} / n)$.

Lemma 1. Let $h: \mathbb{R} \rightarrow \mathbb{R}, d \geq 1$, be a function such that $\int_{\mathbb{R}^{d}} h(\|x\|) e^{-\|x\|^{2}} d x<$ $\infty, h$ is absolutely continuous, and $h^{\prime}$ exists a.e. Let $x_{i} \in \mathbb{R}^{d}, i=1 \ldots, n$ be any points, $n \geq 1$. Then

$$
\int_{\mathbb{R}^{d}} h(\|x\|) \frac{e^{-\|x\|^{2} / 2}}{(2 \pi)^{d / 2}} d x-\frac{1}{n} \sum_{i=1}^{n} h\left(\left\|x_{i}\right\|\right)=\int_{0}^{\infty} R_{\mu}(r) h^{\prime}(r) d r,
$$

where $\mu$ is defined in (4) and $R_{\mu}(r)=\frac{1}{n} \sum_{i=1}^{n} 1_{[0, r)}\left(\left\|x_{i}\right\|\right)-\mu(r), r \in \mathbb{R}_{+}$.

Proof. Niederreiter [6] exhibits the error of a quasi-Monte Carlo method approximating the integral of a differentiable function. We apply a similar technique. For $n, d \geq 1$ consider $x_{i} \in \mathbb{R}^{d}, i=1, \ldots, n$. Then

$$
\begin{array}{rl}
\int_{\mathbb{R}^{d}} & h(\|x\|) \frac{e^{-\|x\|^{2} / 2}}{(2 \pi)^{d / 2}} d x-\frac{1}{n} \sum_{i=1}^{n} h\left(\left\|x_{i}\right\|\right) \\
& =\int_{0}^{\infty} h(r) \mu^{\prime}(r) d r-\frac{1}{n} \sum_{i=1}^{n} h\left(\left\|x_{i}\right\|\right) \\
& =\int_{0}^{1} h\left(\mu^{-1}(t)\right) d t-\frac{1}{n} \sum_{i=1}^{n} h\left(\left\|x_{i}\right\|\right) \\
& =\int_{0}^{1} g(t) d t-\frac{1}{n} \sum_{i=1}^{n} g\left(s_{i}\right), s_{i}=\mu\left(\left\|x_{i}\right\|\right), g=h \circ \mu^{-1} \\
& =\int_{0}^{1} R(t) g^{\prime}(t) d t, R(t)=\frac{1}{n} \sum_{i=1}^{n} 1_{[0, t)}\left(s_{i}\right)-t \\
& =\left.\int_{0}^{1} R(t) \frac{d h(z)}{d z}\right|_{z=\mu^{-1}(t)}\left(\mu^{-1}\right)^{\prime}(t) d t \\
& =\left.\int_{0}^{1} R(t) \frac{d h(z)}{d z}\right|_{z=\mu^{-1}(t)} d \mu^{-1}(t) \\
& =\int_{0}^{\infty} R(\mu(r)) h^{\prime}(r) d r \\
& =\int_{0}^{\infty} R(r) h^{\prime}(r) d r,
\end{array}
$$

which completes the proof.

In the proof of the Lemma 1 we have used the quantity $R_{\mu}(r)=R(\mu(r)), r \in \mathbb{R}_{+}$, which is bounded from above by the discrepancy $D_{n}$ of the points $\mu\left(\left\|x_{i}\right\|\right), i=$ $1, \ldots, n$. This suggests that good sample points can be obtained by appropriately transforming one-dimensional low discrepancy sequences as we will see below.

We now turn our attention to the integral $I_{d}$. For each $f \in F$, we define the error of the method $I_{d, n}(f)$ by

$$
e\left(I_{d, n}, f\right)=\left|I_{d}(f)-I_{d, n}(f)\right| .
$$


We also define the worst case error of this method in the class $F$ by

$$
e\left(I_{d, n}\right)=\sup _{f \in F} e\left(I_{d, n}, f\right) .
$$

Accordingly, the quantity $e\left(I_{d, 1}, f\right)$ is the error we obtain using a sample of size 1 . This quantity can also be viewed as the initial error of the method $I_{d, n}(f)$ when $n>1$.

Lemma 2. For $f \in F$, the method $I_{d, n}$ approximates the integral $I_{d}$ with error

$$
e\left(I_{d, n}, f\right)=\frac{\pi^{d / 2}}{\sqrt{2}}\left|\int_{\mathbb{R}_{+}} R_{\mu}(r) f^{\prime}(r / \sqrt{2}) d r\right|,
$$

where $\mu$ is defined in (4), $R_{\mu}(r)=\frac{1}{n} \sum_{i=1}^{n} 1_{[0, r)}\left(\left\|x_{i}\right\|\right)-\mu(r)$, and $x_{i} \in \mathbb{R}^{d}, i=$ $1, \ldots, n$ are arbitrary but fixed sample points.

Proof. The proof follows from Lemma 1 by setting $h(\cdot)=\pi^{d / 2} f(\cdot / \sqrt{2})$.

Theorem 1. For the class of functions $F$, the error of the method (3) satisfies

$$
e\left(I_{d, n}\right)=\frac{e\left(I_{d, 1}\right)}{\rho\left(\left\|x_{1}\right\|\right)} \int_{\mathbb{R}_{+}}\left|R_{\mu}(r)\right| d r
$$

where $\rho\left(\left\|x_{1}\right\|\right)=\int_{\mathbb{R}_{+}}\left|1_{[0, r)}\left(\left\|x_{1}\right\|\right)-\mu(r)\right| d r$, and $x_{i} \in \mathbb{R}^{d}, i=1, \ldots, n$ are arbitrary but fixed sample points.

Proof. Using Lemma 2 and by considering a function $f \in F$ such that $\left|f^{\prime}\right|=M$ and $R_{\mu}(r) f^{\prime}(r / \sqrt{2}) \geq 0$ we derive $e\left(I_{d, n}\right)$. Similarly, we derive $e\left(I_{d, 1}\right)$ and the proof follows.

We proceed to obtain bounds for $1-\mu(r), r>0$, on which the value of the quantity $\int_{\mathbb{R}_{+}}\left|R_{\mu}(r)\right| d r$ depends. We have

$$
\begin{aligned}
1-\mu(r) & =\int_{r}^{\infty} \mu^{\prime}(y) d y \\
& =\int_{0}^{\infty} \mu^{\prime}(r+y) d y=c_{d}(2 \pi)^{-d / 2} \int_{0}^{\infty}(r+y)^{d-1} e^{-(r+y)^{2} / 2} d y \\
& =\gamma_{d} r^{d-1} e^{-r^{2} / 2} \int_{0}^{\infty}\left(\frac{y}{r}+1\right)^{d-1} e^{-r y} e^{-y^{2} / 2} d y, \quad \gamma_{d}=c_{d}(2 \pi)^{-d / 2} \\
& \leq \gamma_{d} r^{d-1} e^{-r^{2} / 2} \int_{0}^{\infty} e^{-w y} e^{-y^{2} / 2} d y, \quad w=r-\frac{d-1}{r}, \text { and } r^{2} \geq d \geq 1 \\
& \leq \gamma_{d} r^{d-1} e^{-r^{2} / 2} w^{-1},
\end{aligned}
$$

where the last inequality holds by virtue of the fact that $\int_{0}^{\infty} e^{-y z} d y=z^{-1}, z>0$, and $e^{-y^{2} / 2} \leq 1$. Since $r^{2} \geq d \geq 1$, we conclude that $w^{-1} \leq d / r$ (for $d=1$ the above expression holds for $w=r \geq 1$ ) and that

$$
1-\mu(r) \leq d \gamma_{d} r^{d-2} e^{-r^{2} / 2}=d \frac{\mu^{\prime}(r)}{r}, r \geq \sqrt{d} \geq 1 .
$$


In a similar way we derive a lower bound for $1-\mu(r)$. We have

$$
\begin{aligned}
1-\mu(r) & =\gamma_{d} r^{d-1} e^{-r^{2} / 2} \int_{0}^{\infty}\left(\frac{y}{r}+1\right)^{d-1} e^{-y r} e^{-y^{2} / 2} d y \\
& \geq \gamma_{d} r^{d-1} e^{-r^{2} / 2} \int_{0}^{\infty} e^{-y r} e^{-y^{2} / 2} d y \\
& \geq \gamma_{d} r^{d-1} e^{-r^{2} / 2}\left(\frac{1}{r}-\frac{1}{r^{3}}\right), r>0
\end{aligned}
$$

where the last inequality can be found in [16, p. 174].

Theorem 2. There exist deterministic points $x_{i} \in \mathbb{R}^{d}, i=1, \ldots, n$, for which the error of the method $I_{d, n}$ for the integral $I_{d}$ is bounded as follows:

$$
e\left(I_{d, n}\right) \leq \frac{e\left(I_{d, 1}\right)}{\rho(\zeta)} \frac{c}{n}\left[\sqrt{2 \log \left(\frac{d}{\Gamma(d / 2)} n\right)}+d\right](1+o(1)),
$$

where $c$ is a constant, $\rho(\zeta)=\int_{\mathbb{R}_{+}}\left|1_{[0, r)}(\zeta)-\mu(r)\right| d r$, and $\zeta=\mu^{-1}(1 / 2)$, i.e., $e\left(I_{d, n}\right)=O(\sqrt{\log n} / n)$.

Proof. Let $t_{i} \in[0,1), i=1, \ldots, n$, be $n$ numbers with discrepancy $D_{n}=c / n$, where $c \geq 1 / 2$ is a constant. For instance, these numbers can be $n$ terms of a low discrepancy sequence or a $(t, m, 1)$-net. It is shown in [6] that the discrepancy of these points is given by

$$
D_{n}=\frac{1}{2 n}+\max _{1 \leq i \leq n}\left|t_{(i)}-\frac{2 i-1}{2 n}\right|,
$$

where $t_{(1)} \leq \cdots \leq t_{(i)} \leq \cdots \leq t_{(n)}$ denotes the ordered sequence of the points.

This implies that the discrepancy of the sequence

$$
\tau_{i}=\left\{\begin{array}{cl}
t_{i} & \text { if } t_{i}<1-(4 n)^{-1} \\
t_{i}-(4 n)^{-1} & \text { otherwise }
\end{array}, i=1, \ldots, n,\right.
$$

cannot exceed $c / n$ and its maximum term satisfies $\tau_{(n)}<1-(4 n)^{-1}$. Hence, without loss of generality, we assume that $t_{(n)}<1-(4 n)^{-1}$.

Consider $x_{i} \in \mathbb{R}^{d}$ such that $\mu\left(\left\|x_{i}\right\|\right)=t_{i}, i=1, \ldots, n$. Let $r^{*}=r^{*}(n)=$ $\max _{1 \leq i \leq n}\left\{\left\|x_{i}\right\|\right\}$, assume that $n$ is sufficiently large so that $r^{* 2}>d \geq 1$ and consider the error equation of Theorem 1 . We have

$$
\frac{e\left(I_{d, n}\right)}{e\left(I_{d, 1}\right)}=\frac{1}{\rho\left(\left\|x_{1}\right\|\right)} \int_{\mathbb{R}_{+}}\left|R_{\mu}(r)\right| d r \leq \frac{1}{\rho(\zeta)} \int_{\mathbb{R}_{+}}\left|R_{\mu}(r)\right| d r,
$$

because $\rho(s) \geq \rho(\zeta), \forall s \in \mathbb{R}_{+}$, for $\zeta=\mu^{-1}(1 / 2)$. Thus,

$$
\rho(\zeta) \frac{e\left(I_{d, n}\right)}{e\left(I_{d, 1}\right)} \leq \int_{0}^{r^{*}}\left|R_{\mu}(r)\right| d r+\int_{r^{*}}^{\infty}[1-\mu(r)] d r .
$$

Since $\left|R_{\mu}(r)\right| \leq c / n, r \in \mathbb{R}_{+}$, we estimate the first term of the above equation by $c r^{*} / n$. We estimate the second term using (5) and

$$
\int_{r}^{\infty}\left|R_{\mu}(z)\right| d z \leq \int_{r}^{\infty}[1-\mu(z)] d z \leq d[1-\mu(r)], r \geq \sqrt{d} \geq 1
$$

and $1-\mu\left(r^{*}\right) \leq c / n$. Thus,

$$
\rho(\zeta) \frac{e\left(I_{d, n}\right)}{e\left(I_{d, 1}\right)} \leq \frac{c}{n}\left(r^{*}+d\right) .
$$


Consider the function

$$
q=g(r)=d \frac{\mu^{\prime}(r)}{r}=d \gamma_{d} r^{d-2} e^{-r^{2} / 2}, r>\sqrt{d} \geq 1 .
$$

We estimate its inverse, $g^{-1}$, by

$$
h(q)=\left\{2\left[\log \left(a_{d} q^{-1}\right)+\frac{d-2}{2} \log \log \left(a_{d} q^{-1}\right)\right]\right\}^{1 / 2}=\sqrt{2 \log \left(a_{d} q^{-1}\right)}(1+o(1))
$$

where $a_{d}=d / \Gamma(d / 2)$. Indeed,

$$
\begin{aligned}
g(h(q))= & \frac{d \gamma_{d} 2^{(d-2) / 2}}{a_{d}}\left\{\log \left(a_{d} q^{-1}\right)+\frac{d-2}{2} \log \log \left(a_{d} q^{-1}\right)\right\}^{(d-2) / 2} \\
& \times \frac{q}{\left[\log \left(a_{d} q^{-1}\right)\right]^{(d-2) / 2}} \\
= & q(1+o(1)), \text { as } q \rightarrow 0 .
\end{aligned}
$$

From (5) and (6) we have tight bounds for $\mu(r)$,

$$
\frac{1}{d} g(r)\left(1-\frac{1}{d}\right) \leq \frac{1}{d} g(r)\left[1-\frac{1}{r^{2}}\right] \leq 1-\mu(r) \leq g(r), \quad r>\sqrt{d} \geq 1 .
$$

The function $g$ is decreasing for $r^{2}>d-2$, which implies that $g^{-1}\left[1-\mu\left(r^{*}\right)\right] \geq r^{*}$. We substitute $r^{*}$ by the value $g^{-1}\left[1-\mu\left(r^{*}\right)\right]$ in the error estimate to obtain

$$
\rho(\zeta) \frac{e\left(I_{d, n}\right)}{e\left(I_{d, 1}\right)} \leq \frac{c}{n}\left(g^{-1}\left[1-\mu\left(r^{*}\right)\right]+d\right) .
$$

Since we have assumed that $t_{(n)}<1-(4 n)^{-1}$ and $\mu\left(r^{*}\right)=t_{(n)}$, we have $1-$ $\mu\left(r^{*}\right)>(4 n)^{-1}$. This implies that $r=g^{-1}\left[1-\mu\left(r^{*}\right)\right]=\sqrt{2 \log \left(a_{d} n\right)}(1+o(1))$, which completes the proof.

We do not know if the bound of Theorem 2 is sharp. The proof of Theorem 2 not only shows how good sample points can be obtained but also how the quality of any sample can be assessed by calculating the discrepancy of its points. If the discrepancy $D_{\mu, n}$ of the sample points $x_{i}, i=1, \ldots, n$, is small, then the error of the method $I_{d, n}$ will be small.

The quantity $\sqrt{2 \log \left(a_{d} n\right)}, a_{d}=d / \Gamma(d / 2)$, in the error bound of Theorem 2 is small in practice. For $d$ as small as 6 a sample of size $n>10^{8}$ is required for $\sqrt{2 \log \left(a_{d} n\right)}>d$.

Corollary 1. For $\sqrt{2 \log \left(a_{d} n\right)} \leq d, a_{d}=d / \Gamma(d / 2)$, we have

$$
e\left(I_{d, n}\right) \leq \frac{e\left(I_{d, 1}\right)}{\rho(\zeta)} 2 d \frac{c}{n}
$$

Corollary 2. If $r^{*}$ is small so that $r^{*}=r^{*}(n) \leq \sqrt{d}$, then we have

$$
e\left(I_{d, n}\right) \leq \frac{e\left(I_{d, 1}\right)}{\rho(\zeta)}[\sqrt{d}+d] \frac{c}{n} .
$$


Proof. When $r^{*} \leq \sqrt{d}$ we cannot directly use (5) as we did in the proof of Theorem 2. Using $\left|R_{\mu}(r)\right| \leq c / n, r \in \mathbb{R}_{+}$, we have

$$
\begin{aligned}
\int_{0}^{\infty}\left|R_{\mu}(r)\right| d r & \leq \int_{0}^{\sqrt{d}}\left|R_{\mu}(r)\right| d r+\int_{\sqrt{d}}^{\infty}[1-\mu(r)] d r \\
& \leq \frac{c \sqrt{d}}{n}+d[1-\mu(\sqrt{d})] \leq \frac{c \sqrt{d}}{n}+d\left[1-\mu\left(r^{*}\right)\right] \\
& \leq \frac{c}{n}[\sqrt{d}+d],
\end{aligned}
$$

which completes the proof.

Corollaries 1 and 2 show conditions that relate the size of the dimension $d$ and the sample size $n$ and how these conditions affect the error of the method $I_{d, n}$. These conditions can be interesting in practice.

In all cases, the size of $D_{\mu, n}$ and the conditions of the above two corollaries can be easily checked to yield practical numerical error estimates. For instance, the discrepancy of the points used by QMC-GF in [11] is small for $n \leq 10^{6}$ and $d \leq 100$. This implies that they can be used to efficiently evaluate integrals of functions in the class $F$. Thus, simulation results reporting fast convergence even when $d$ is large as in 9, 11] can be explained.

\section{ACKNOWLEDGMENTS}

I wish to thank F. Curbera, S. Tezuka, J. Traub and G. Wasilkowski for their comments which greatly improved this paper.

\section{REFERENCES}

[1] Bratley, P., Fox, B.L., and Niederreiter, H. (1992), Implementation and Tests of LowDiscrepancy Sequences, ACM Trans. on Modeling and Computer Simulation, 2:3, 195-213.

[2] Capstick, S., and Keister, B.D. (1996), Multidimensional quadrature algorithms at higher degree and/or dimension, Journal of Computational Physics, 123, 267-273. CMP 96:07

[3] Drmota, M., Tichy, R.F. (1997), "Sequences, discrepancies and applications," Lecture Notes in Mathematics, 1651, Springer, New York. MR 98j:11057

[4] Joy, C., Boyle, P.P., and Tan, K.S. (1996), Quasi-Monte Carlo Methods in Numerical Finance, Management Science, 42, No. 6, 926-938.

[5] Keister, B.D. (1996), Multidimensional Quadrature Algorithms, Computers in Physics, 10:20, $119-122$.

[6] Niederreiter, H. (1992), "Random Number Generation and Quasi-Monte Carlo Methods," CBMS-NSF Regional Conference Series in Applied Math. No. 63, SIAM. MR 93h:65008

[7] Ninomiya, S., and Tezuka, S. (1996), Toward real-time pricing of complex financial derivatives, Applied Mathematical Finance, 3, 1-20.

[8] Novak, E., Ritter, K. (1996), High dimensional integration of smooth functions over cubes, Numer. Math., 75, 79-97. MR 97k:65057

[9] Novak, E., Ritter, K., Schmitt, R., Steinbauer, A. (1997), On a recent interpolatory method for high dimensional integration, Preprint University of Erlangen.

[10] Papageorgiou, A., and Traub, J.F. (1996), Beating Monte Carlo, Risk, 9:6, 63-65.

[11] Papageorgiou, A., and Traub, J.F. (1997), Faster evaluation of multi-dimensional integrals, Computers in Physics, Nov./Dec., 574-578.

[12] Paskov, S.H. and Traub, J.F., Faster Valuation of Financial Derivatives, Journal of Portfolio Management, Fall, 1995, 113-120.

[13] Paskov, S.H. (1997), New Methodologies for Valuing Derivatives, in "Mathematics of Derivative Securities," S. Pliska and M. Dempster eds., Isaac Newton Institute, Cambridge University Press, Cambridge, UK, 545-582. CMP 98:06 
[14] Sloan, I.H., and Woźniakowski, H. (1998), When Are Quasi-Monte Carlo Algorithms Efficient for High Dimensional Integrals?, J. Complexity, 14(1), 1-33.

[15] Tezuka, S. (1995), "Uniform Random Numbers: Theory and Practice," Kluwer Academic Publishers, Boston.

[16] Tong, Y.L. (1990), "The Multivariate Normal Distribution," Springer Verlag, New York. MR 91g:60021

[17] Traub, J.F. and Werschulz, A.G. (1998), "Complexity and Information," Cambridge University Press, Cambridge, UK. CMP 99:13

[18] Woźniakowski, H. (1991), Average case complexity of multivariate integration, Bulletin of the American Mathematical Society, 24, 185-194. MR 91i:65224.

Department of Computer Science, Columbia University, New York, NY 10027

E-mail address: ap@cs.columbia.edu 